PROCEEDINGS OF THE

AMERICAN MATHEMATICAL SOCIETY

Volume 139, Number 12, December 2011, Pages 4193-4197

S 0002-9939(2011)10897-9

Article electronically published on April 20, 2011

\title{
AN UPPER BOUND ON THE NUMBER OF $F$-JUMPING COEFFICIENTS OF A PRINCIPAL IDEAL
}

\author{
MORDECHAI KATZMAN, GENNADY LYUBEZNIK, AND WENLIANG ZHANG
}

(Communicated by Harm Derksen)

\begin{abstract}
We prove a result relating the Jacobian ideal and the generalized test ideal associated to a principal ideal in $R=k\left[x_{1}, \ldots, x_{n}\right]$ with $\left[k: k^{p}\right]<\infty$ or in $R=k\left[\left[x_{1}, \ldots, x_{n}\right]\right]$ with an arbitrary field $k$ of characteristic $p>0$. As a consequence of this result, we establish an upper bound on the number of $F$-jumping coefficients of a principal ideal with an isolated singularity.
\end{abstract}

\section{INTRODUCTION}

In characteristic zero, one can define invariants, called jumping coefficients in ELSV04, attached to an ideal sheaf on a smooth variety via multiplier ideals. These jumping coefficients are positive rational numbers which encode interesting geometric and algebraic information (see [Laz04, Chapter 9] for details). In ELSV04 the following connection between Jacobian and multiplier ideals is established.

Theorem 1.1 (Proposition 3.11 in [ELSV04]). Given $f \in \mathbb{C}\left[x_{1}, \ldots, x_{n}\right]$, one has

$$
\operatorname{Jac}(f) \subseteq \mathcal{J}\left((f)^{1-\epsilon}\right), \text { for all } \epsilon>0,
$$

where $\operatorname{Jac}(f)=\left(f, \frac{\partial f}{\partial x_{1}}, \ldots, \frac{\partial f}{\partial x_{n}}\right)$ and $\mathcal{J}\left((f)^{1-\epsilon}\right)$ is the multiplier ideal of the pair $\left(\mathbb{C}\left[x_{1}, \ldots, x_{n}\right],(f)^{1-\epsilon}\right)$.

As a consequence, one has

Corollary 1.2. If $f \in R=\mathbb{C}\left[x_{1}, \ldots, x_{n}\right]$ has an isolated singularity, then $f$ has at most $\operatorname{dim}_{\mathbb{C}}\left(\frac{R}{\operatorname{Jac}(f)}\right)+1$ jumping coefficients in $[0,1]$.

The purpose of this paper is to extend these results to characteristic $p>0$.

In characteristic $p>0$, Hara and Yoshida introduced, in [HY03, an analogue of the multiplier ideals, namely, the generalized test ideals. Generalized test ideals are defined in any noetherian ring of characteristic $p>0$, but the definition is less technical when the ring $R$ is an $F$-finite regular ring or an excellent regular local ring. When $R$ is an $F$-finite regular ring (see [BMS08]) or an excellent regular local ring (see [KLZ09]), for each ideal $J$ of $R$ and each positive integer $e$, there exists a

Received by the editors October 14, 2010.

2010 Mathematics Subject Classification. Primary 13A35, 13N10.

Key words and phrases. $F$-jumping coefficient, test ideal, Jacobian ideal.

${ }^{1}$ In the statement of [ELSV04 Proposition 3.8], $f$ is assumed to have an isolated singularity. However, this assumption is not needed in the proof of the statement.

(C)2011 American Mathematical Society 
unique smallest ideal $I_{e}(J)$ such that $J \subseteq\left(I_{e}(J)\right)^{\left[p^{e}\right]}$. Then, for each nonnegative real number $t$, the generalized test ideal, $\tau\left(J^{t}\right)$, can be defined as

$$
\bigcup_{e} I_{e}\left(J^{\left\lceil t p^{e}\right\rceil}\right)
$$

In this context, we say that $c$ is an F-jumping coefficient of $J$ if $\tau\left(J^{c}\right) \varsubsetneqq \tau\left(J^{c^{\prime}}\right)$ for all $c^{\prime}<c$. It is proved, in BMS09, when $R$ is an $F$-finite regular ring and in KLZ09] when $R$ is an excellent regular local ring, that $F$-jumping coefficients of each principal ideal of $R$ consist of a discrete set of positive rational numbers.

Let $R=k\left[x_{1}, \ldots, x_{n}\right]$ with $\left[k: k^{p}\right]<\infty$ or $R=k\left[\left[x_{1}, \ldots, x_{n}\right]\right]$ with $k$ an arbitrary field of characteristic $p>0$. Let $\frac{\partial f}{\partial x_{i}}$ denote the partial derivative of $f \in R$ with respect to $x_{i}$ and let $\operatorname{Jac}(f)=\left(f, \frac{\partial f}{\partial x_{1}}, \ldots, \frac{\partial f}{\partial x_{n}}\right)$. Our main theorem of this paper is the following analogue of Theorem 1.1 .

Theorem 1.3 (Main Theorem). Let $R$ be as above. Then

$$
\operatorname{Jac}(f) \subseteq \tau\left((f)^{1-\epsilon}\right),
$$

for all $\epsilon>0$.

When $k$ is a perfect field of characteristic $p>0$, the singular locus of $R /(f)$ is determined by $\operatorname{Jac}(f)$. In particular, when $f$ (or equivalently $R /(f)$ ) has an isolated singularity, $\operatorname{dim}_{k}\left(\frac{R}{\operatorname{Jac}(f)}\right)$ is finite. Note that in this case every chain of ideals between $R$ and $\operatorname{Jac}(f)$ has length at $\operatorname{most}_{k} \operatorname{dim}_{k}\left(\frac{R}{\operatorname{Jac}(f)}\right)$. Therefore, as a consequence of our Main Theorem, we have

Corollary 1.4. Let $k$ be a perfect field of characteristic $p>0$, and let $R=$ $k\left[x_{1}, \ldots, x_{n}\right]$ or $k\left[\left[x_{1}, \ldots, x_{n}\right]\right]$. Then, for each $f \in R$ with isolated singularity, there are at most $\operatorname{dim}_{k}\left(\frac{R}{\operatorname{Jac}(f)}\right)+1$ F-jumping coefficients of $f$ in $[0,1]$.

\section{A Result on Differential operators}

In this section we consider differential operators over $R=\mathbb{Z}\left[x_{1}, \ldots, x_{n}\right]$ (or $R=$ $\mathbb{Z}\left[\left[x_{1}, \ldots, x_{n}\right]\right]$, respectively): let $D_{m, i}: R \rightarrow R$ be the $\mathbb{Z}\left[x_{1}, \ldots, x_{i-1}, x_{i+1}, \ldots, x_{n}\right]$ linear (or $\mathbb{Z}\left[\left[x_{1}, \ldots, x_{i-1}, x_{i+1}, \ldots, x_{n}\right]\right]$-linear, respectively) map that sends $x_{i}^{\ell}$ to $\left(\begin{array}{c}\ell \\ m\end{array}\right) x_{i}^{\ell-m}$ and let $D_{0, i}$ be the identity map. We may write $D_{m, i}$ as

$$
D_{m, i}=\frac{1}{m !} \frac{\partial^{m}}{\partial x_{i}^{m}}: R \rightarrow R .
$$

Even though the following proposition is stated over a field $k$ in [Lyu10, the same proof works over $\mathbb{Z}$.

Proposition 2.1 (Proposition 2.1 in Lyu10). For each $f \in R$, we have

$$
D_{m, i} \cdot f=\sum_{\ell=0}^{m} D_{\ell, i}(f) \cdot D_{m-\ell, i} \text { in } \operatorname{End}_{\mathbb{Z}}(R) ;
$$

i.e., given $f, g \in R$, we have

$$
D_{m, i}(f g)=\sum_{\ell=0}^{m} D_{\ell, i}(f) D_{m-\ell, i}(g) \text { in } R .
$$

Our main result of this section is the following identity. 
Theorem 2.2. Given any $f \in R$ and a positive integer $m$, we have

$$
\sum_{\ell=0}^{m}(\ell-1) D_{\ell, i}(f) D_{m-\ell, i}\left(f^{m-1}\right)=0 \text {. }
$$

Proof. Writing $D_{m, i}$ as $\frac{1}{m !} \frac{\partial^{m}}{\partial x_{i}^{m}}$ and multiplying (11) by $m$ !, we can rewrite (1) as

$$
\sum_{\ell=0}^{m}(\ell-1)\left(\begin{array}{c}
m \\
\ell
\end{array}\right) \frac{\partial^{\ell} f}{\partial x_{i}^{\ell}} \frac{\partial^{m-\ell} f^{m-1}}{\partial x_{i}^{m-\ell}}=0 .
$$

To ease our notation, we will write $\frac{\partial^{\ell} f}{\partial x_{i}^{\ell}}$ as $\partial^{\ell}(f)$ and $\frac{\partial^{m-\ell} f^{m-1}}{\partial x_{i}^{m-\ell}}$ as $\partial^{m-\ell}\left(f^{m-1}\right)$. Hence the above equation becomes

$$
\sum_{\ell=0}^{m}(\ell-1)\left(\begin{array}{c}
m \\
\ell
\end{array}\right) \partial^{\ell}(f) \partial^{m-\ell}\left(f^{m-1}\right)=0 .
$$

We first expand

$$
\sum_{\ell=0}^{m}(\ell-1)\left(\begin{array}{c}
m \\
\ell
\end{array}\right) \partial^{\ell}(f) \partial^{m-\ell}\left(f^{m-1}\right)
$$

as

(3) $-f \partial^{m}\left(f^{m-1}\right)-\sum_{\ell=2}^{m}\left(\begin{array}{c}m \\ \ell\end{array}\right) \partial^{\ell}(f) \partial^{m-\ell}\left(f^{m-1}\right)+\sum_{\ell=2}^{m} \ell\left(\begin{array}{c}m \\ \ell\end{array}\right) \partial^{\ell}(f) \partial^{m-\ell}\left(f^{m-1}\right)$.

Use the fact that $\ell\left(\begin{array}{c}m \\ \ell\end{array}\right)=m\left(\begin{array}{c}m-1 \\ \ell-1\end{array}\right)$ to rewrite (3) as

$$
-f \partial^{m}\left(f^{m-1}\right)-\sum_{\ell=2}^{m}\left(\begin{array}{c}
m \\
\ell
\end{array}\right) \partial^{\ell}(f) \partial^{m-\ell}\left(f^{m-1}\right)+\sum_{\ell=2}^{m} m\left(\begin{array}{c}
m-1 \\
\ell-1
\end{array}\right) \partial^{\ell}(f) \partial^{m-\ell}\left(f^{m-1}\right) .
$$

Note that Leibniz's rule implies that

$$
\partial^{m}\left(f^{m}\right)=\partial^{m}\left(f f^{m-1}\right)=\sum_{l=0}^{m}\left(\begin{array}{c}
m \\
l
\end{array}\right) \partial^{l}(f) \partial^{m-l}\left(f^{m-1}\right)
$$

and rewrite (4) as

$$
\begin{array}{r}
-f \partial^{m}\left(f^{m-1}\right)-\left(\partial^{m}\left(f^{m}\right)-f \partial^{m}\left(f^{m-1}\right)-m \partial(f) \partial^{m-1}\left(f^{m-1}\right)\right) \\
+\sum_{\ell=1}^{m-1} m\left(\begin{array}{c}
m-1 \\
\ell
\end{array}\right) \partial^{\ell}(\partial(f)) \partial^{m-1-\ell}\left(f^{m-1}\right) .
\end{array}
$$

Now

$$
\begin{aligned}
& m \partial(f) \partial^{m-1}\left(f^{m-1}\right)+\sum_{\ell=1}^{m-1} m\left(\begin{array}{c}
m-1 \\
\ell
\end{array}\right) \partial^{\ell}(\partial(f)) \partial^{m-1-\ell}\left(f^{m-1}\right) \\
& =m \sum_{\ell=0}^{m-1}\left(\begin{array}{c}
m-1 \\
\ell
\end{array}\right) \partial^{\ell}(\partial(f)) \partial^{m-1-\ell}\left(f^{m-1}\right) \\
& =\partial^{m-1}\left(m(\partial f) f^{m-1}\right) \\
& =\partial^{m-1} \partial f^{m}=\partial^{m} f^{m},
\end{aligned}
$$

and (5) simplifies to

$$
-f \partial^{m}\left(f^{m-1}\right)-\partial^{m}\left(f^{m}\right)+f \partial^{m}\left(f^{m-1}\right)+\partial^{m} f^{m}=0 .
$$




\section{Proof of the Main Theorem}

Throughout this section, $R$ is either $k\left[x_{1}, \ldots, x_{n}\right]$ with $\left[k: k^{p}\right]<\infty$ or $k\left[\left[x_{1}, \ldots, x_{n}\right]\right]$ with $k$ an arbitrary field of characteristic $p>0$. In either case, by reducing the operators $D_{m, i} \bmod p$, we get differential operators over $k$, which will still be denoted $D_{m, i}$. Note that the identity (11) also holds over $k$ and that each differential operator $D_{m, i}$ is $R^{p^{e}}$-linear when $m<p^{e}$.

We begin with an easy observation.

Lemma 3.1. Given any $f \in R$, we have

$$
D_{p^{e}, i}\left(f^{p^{e}}\right)=\left(\frac{\partial f}{\partial x_{i}}\right)^{p^{e}}
$$

Proof. Since $D_{p^{e}, i}$ is $k$-linear and $f$ is a $k$-linear combination of monomials, it suffices to consider the case when $f$ is a monomial. Also, since $D_{p^{e}, i}$ is $k\left[x_{1}, \ldots\right.$, $\left.\hat{x}_{i}, \ldots, x_{n}\right]$-linear (or $k\left[\left[x_{1}, \ldots, \hat{x}_{i}, \ldots, x_{n}\right]\right]$-linear), it suffices to consider the case when $f=x_{i}^{t}$ for some $t \in \mathbb{N}$. We have

$$
D_{p^{e}, i}\left(f^{p^{e}}\right)=\left(\begin{array}{c}
t p^{e} \\
p^{e}
\end{array}\right) x_{i}^{(t-1) p^{e}} \text { and }\left(\frac{\partial f}{\partial x_{i}}\right)^{p^{e}}=t^{p^{e}} x_{i}^{(t-1) p^{e}} .
$$

But it is well-known that $\left(\begin{array}{c}t p^{e} \\ p^{e}\end{array}\right) \equiv t(\bmod p)$ (cf. Eis95, Lemma 15.22 (Lucas's Theorem)]) and Fermat's Little Theorem implies that $t^{p^{e}} \equiv t(\bmod p)$.

We can now prove our Main Theorem.

Proof of Main Theorem. Clearly it is enough to prove that

$$
\operatorname{Jac}(f) \subseteq \tau\left((f)^{1-\frac{1}{p^{e}}}\right)
$$

for all integers $e>0$.

Using the definition of $\tau\left((f)^{1-\frac{1}{p^{e}}}\right)$ and the fact that $R$ is noetherian, we can write $\tau\left((f)^{1-\frac{1}{p^{e}}}\right)=I_{e^{\prime}}\left((f)^{\left\lceil p^{e^{\prime}}\left(1-\frac{1}{p^{e}}\right)\right\rceil}\right)=I_{\left(e^{\prime}-e\right)+e}\left((f)^{p^{e^{\prime}-e}\left(p^{e}-1\right)}\right)$ for some $e^{\prime} \geq e$ and BMS08] [Lemma 2.4(4)] implies that this equals $I_{e}\left((f)^{p^{e}-1}\right)$.

Thus $\tau\left((f)^{1-\frac{1}{p^{e}}}\right)=I_{e}\left(f^{p^{e}-1}\right)$ is the smallest ideal $J$ such that $f^{p^{e}-1} \subseteq J^{\left[p^{e}\right]}$, so it suffices to prove that for any ideal $J$ with $f^{p^{e}-1} \subseteq J^{\left[p^{e}\right]}$, we have

$$
\frac{\partial f}{\partial x_{i}} \in J
$$

Since $D_{t, i}$ with $t<p^{e}$ is $R^{p^{e}}$-linear, we have

$$
D_{t, i}\left(f^{p^{e}-1}\right) \in J^{\left[p^{e}\right]} \text {, for all } t<p^{e} .
$$

Setting $m=p^{e}$ in the identity (11), we have that

$$
f D_{p^{e}, i}\left(f^{p^{e}-1}\right)=\sum_{\ell=2}^{p^{e}}(\ell-1) D_{\ell, i}(f) D_{p^{e}-\ell, i}\left(f^{p^{e}-1}\right) \in J^{\left[p^{e}\right]} .
$$

According to Proposition 2.1.

$$
D_{p^{e}, i}\left(f^{p^{e}}\right)=D_{p^{e}, i}\left(f f^{p^{e}-1}\right)=\sum_{\ell=0}^{p^{e}} D_{\ell, i}(f) D_{p^{e}-\ell, i}\left(f^{p^{e}-1}\right),
$$


and hence $D_{p^{e}, i}\left(f^{p^{e}}\right) \in J^{\left[p^{e}\right]}$. Combining this with Lemma 3.1, we see that

$$
\left(\frac{\partial f}{\partial x_{i}}\right)^{p^{e}} \in J^{\left[p^{e}\right]}
$$

and consequently,

$$
\frac{\partial f}{\partial x_{i}} \in J
$$

since $R$ is regular. This finishes the proof of our Main Theorem.

\section{ACKNOWLEDGMENTS}

The results in this paper were obtained while the first and third authors enjoyed the hospitality of the School of Mathematics at the University of Minnesota. The second author gratefully acknowledges support through NSF grant DMS 0701127. The first author also wishes to acknowledge support through EPSRC grant $\mathrm{EP} / \mathrm{H} 040684 / 1$. This work was initiated at the Commutative Algebra MRC held in June 2010. The third author wishes to thank the organizers for providing a stimulating atmosphere.

\section{REFERENCES}

[BMS08] M. Blickle, M. Mustaţă, and K. E. Smith, Discreteness and rationality of F-thresholds, Michigan Math. J. 57 (2008), 43-61. MR2492440 (2010c:13003)

[BMS09] M. Blickle, M. Mustaţă, and K. E. Smith, F-thresholds of hypersurfaces, Trans. Amer. Math. Soc. 361 (2009), no. 12, 6549-6565. MR2538604 (2011a:13006)

[Eis95] D. Eisenbud, Commutative algebra. With a view toward algebraic geometry. Graduate Texts in Mathematics, 150. Springer-Verlag, New York, 1995. MR.1322960 (97a:13001)

[ELSV04] L. Ein, R. Lazarsfeld, K. E. Smith, and D. Varolin, Jumping coefficients of multiplier ideals, Duke Math. J. 123 (2004), no. 3, 469-506. MR2068967 (2005k:14004)

[HY03] N. Hara and K.-I. Yoshida, A generalization of tight closure and multiplier ideals, Trans. Amer. Math. Soc. 355 (2003), 3143-3174. MR.1974679 (2004i:13003)

[KLZ09] M. Katzman, G. Lyubeznik, and W. Zhang, On the discreteness and rationality of F-jumping coefficients, J. Algebra 322 (2009), no. 9, 3238-3247. Special issue in honor of Paul Roberts. MR2567418

[Laz04] R. Lazarsfeld, Positivity in Algebraic Geometry. I, II, Springer, 2004. MR 2095471 (2005k:14001a) MR2095472(2005k:14001b)

[Lyu10] G. Lyubeznik, A characteristic-free proof of a basic result on $\mathcal{D}$-modules, J. Pure Appl. Algebra 215 (2011), 2019-2023.

Department of Pure Mathematics, University of Sheffield, Hicks Building, Sheffield S3 7RH, United Kingdom

E-mail address: M.Katzman@sheffield.ac.uk

Department of Mathematics, University of Minnesota, Minneapolis, Minnesota 55455

E-mail address: gennady@math.umn.edu

Department of Mathematics, University of Michigan, Ann Arbor, Michigan 48109

E-mail address: wlzhang@umich.edu 